\title{
Attenuated Motor Cortical Responsiveness to Motor and Cognitive Tasks in Generalized Anxiety Disorder
}

\author{
Cheng-Ta Li ${ }^{1,2,3,+, *}$, Chia-Feng Lu ${ }^{4,5+, *}$, Yu-Te Wu${ }^{3}$, Szu-Hui Lee ${ }^{3}$, Ruei-Wen Chu' ${ }^{1}$, Tung-Ping Su ${ }^{1,2,3}$
}

\begin{abstract}
Generalized anxiety disorder (GAD) is characterized by excessive and uncontrollable worries. Recent research revealed impaired intracortical facilitation of bilateral motor cortices (M1) in GAD. Therefore, we aimed to elucidate whether M1 cortical activations could be a reliable and clinically-feasible biomarker for GAD. Forty GAD patients and twenty healthy control subjects were recruited. Bilateral M1 cortical activations in response to 4 different tasks, including finger tapping (FT), verbal fluency test (VFT), dual tasks with simultaneous FT and VFT (DT), and DT with pretreated pressure to enhance worries (DTp), were measured by multichannel functional near-infrared spectroscopy (fNIRS). Task-induced total hemoglobin changes ( $\Delta$ $[\mathrm{Hb}]$ ) were used as the index of cortical activations. The quantitative $\Delta[\mathrm{Hb}]$ results revealed that GAD had abnormally decreased $\Delta[\mathrm{Hb}]$ over bilateral M1. Repeated-measure ANCOVA revealed a significant main effect of group on bilateral $M 1 \Delta[\mathrm{Hb}$ ] results (left M1: $F=11.026$, $p=0.002$; right $\mathrm{M} 1: \mathrm{F}=9.843, p=0.003$ ), indicating that $\mathrm{GAD}$ had significantly lowered $\mathrm{M} 1 \Delta[\mathrm{Hb}]$ across all tasks. The ROC analysis revealed that M1 $\Delta[\mathrm{Hb}]$ in response to VFT demonstrated good sensitivity/specificity in predicting a diagnosis of GAD. In conclusion, attenuated M1 cortical responsiveness to motor and cognitive tasks as a characteristic feature of GAD could have the diagnostic value in clinically settings.
\end{abstract}

\section{Keywords:}

Primary motor cortex, Generalized anxiety disorder, fNIRS, Hemoglobin

\section{Introduction \\ Generalized anxiety disorder (GAD) is a chronic and common mental disorder which is characterized by excessive and uncontrollable worries. Such excessive worrying or anxious apprehension could significantly impair daily functioning and even affect cardiovascular function of an individual [1]. \\ Cortical-amygdala dysregulation is critically involved in the central mechanisms of GAD $[2,3]$. The amygdala is responsible for the}

processing of fear responses [4]. Previous research investing resting-state functional connectivity had indicated that GAD patients had decreased connectivity from several primary and higherorder cortical regions, including prefrontal cortex (PFC) and primary motor cortex (M1), to sub-regions of the amygdala [5]. In addition, activities of $\mathrm{M} 1$ and corticospinal motor responses to magnetic stimulation to $\mathrm{M} 1$ have been found to be changed significantly during worrying, which is a cardinal symptom of GAD [6]. One recent study also indicated that GAD is

\footnotetext{
'Department of Psychiatry, Taipei Veterans General Hospital, Taipei, Taiwan

${ }^{2}$ Division of Psychiatry, Faculty of Medicine, National Yang-Ming University, Taipei, Taiwan

${ }^{3}$ Institute of Brain Science, National Yang-Ming University, Taipei, Taiwan

${ }^{4}$ Translational Imaging Research Center, College of Medicine, Taipei Medical University, Taiwan

${ }^{5}$ Department of Physical Therapy and Assistive Technology, National Yang-Ming University, Taiwan

* Authors Contributed Equally

${ }^{\dagger}$ Author for correspondence: Cheng-Ta Li, MD, PhD, Department of Psychiatry, Taipei Veterans General Hospital, No. 201, Sec. 2, Shih-Pai Road, Beitou district, Taipei 112, Taiwan, Tel: +886-2-28757027 \#298; Fax: +886-2-28757592; email: ctli2@vghtpe.gov.tw
} 
associated with impaired intracortical facilitation in bilateral M1 in a relaxed status [7]. However, reliable biomarkers of GAD are still warranted in the clinical settings.

The present study aimed to find out reliable and clinically-feasible biomarkers of GAD. More specifically, we adopted functional near-infrared spectroscopy (fNIRS) and focused on primary motor cortex (M1) to compare M1 activations in response to tasks specifically designed for testing motor cortical function and provoking anxiety among healthy subjects and GAD patients. The use of multichannel fNIRS was to avoid unnecessary stress during the imaging procedure in GAD patients who were readily anxious and sensitive to environmental changes and to measure cortical activations of $\mathrm{M} 1$ in response to motor and cognitive tasks. fNIRS is a non-invasive brain imaging technique which measures hemoglobin $(\mathrm{Hb})$ concentration changes in the cortical surface area located beneath the detecting probes without the need to place subjects in an uncomfortable or unnatural environment (e.g., staying lying inside a narrow space with head fixation) and without exposure to radiations or acoustic noises [8]. fNIRS has been proven to be a reliable tool for monitoring brain activity and can additionally provide compatible measurement with fMRI data in the investigation of the activation of sensorimotor cortex during motor tasks. We speculated that impaired M1 activation could be a characteristic feature of GAD.

\section{Methods}

\section{- Participants}

This study recruited a total of forty patients with confirmed DSM-IV diagnosis of GAD $(n=40)$, including 20 patients with comorbid diagnosis of major depressive disorder (GAD+MDD) and 20 patients without a comorbidity of MDD (GADMDD) (Table 1). The psychiatric diagnosis was confirmed by the Mini International Neuropsychiatric Interview (MINI) based on the Diagnostic and Statistical Manual of Mental Disorders-IV-Text Revision (DSM-IV-TR) criteria (American Psychiatric Association, 2000). All the GAD patients with or without MDD were drugnaïve or drug-free for at least one week. We also recruited 20 healthy control subjects (HC, $n=20)$ who were free of major medical and neurological illnesses or a history of alcohol or substance abuse. In addition, all healthy control subjects were free from a diagnosis of psychiatric disorder as determined by the MINI. The exclusion

\begin{tabular}{|c|c|c|c|c|c|c|}
\hline & (H) & $\begin{array}{l}\text { GAD-MDD } \\
\text { (G) }\end{array}$ & $\begin{array}{l}\text { GAD+MDD } \\
\text { (M) }\end{array}$ & $\begin{array}{l}\text { GAD vs HC } \\
P\end{array}$ & $\begin{array}{l}3 \text { group } \\
P\end{array}$ & Post-hoc (LSD) \\
\hline Age & $41.8(10.2)$ & $44.3(10.9)$ & $45.3(9.2)$ & 0.187 & 0.209 & \\
\hline Female (\%) & $10(50 \%)$ & $10(50 \%)$ & $10(50 \%)$ & $1.000(x 2)$ & $\begin{array}{l}1.000 \\
(x 2)\end{array}$ & \\
\hline Duration (years) & - & $13.0(10.7)$ & $11.3(11.9)$ & - & 0.639 (t-test) & \\
\hline Go/No-Go Median & $444.6(88.7)$ & 443.9 (122.4) & $420.6(67.3)$ & 0.633 & 0.658 & \\
\hline Go/No-Go S.D. & $71.0(16.1)$ & $81.0(22.7)$ & $73.8(22.2)$ & 0.258 & 0.289 & \\
\hline $\begin{array}{l}\text { 100\% MEP } \\
\text { (left M1) }\end{array}$ & $64.2(9.4)$ & $68.1(12.8)$ & $70.9(13.0)$ & 0.077 & 0.219 & \\
\hline $\begin{array}{l}\text { 100\% MEP } \\
\text { (right M1) }\end{array}$ & $62.0(8.6)$ & 67.5 (12.9) & 68.5 (13.0) & $0.037^{*}$ & 0.178 & \\
\hline HAMD & $0.4(0.9)$ & 7.7 (3.8) & $17.1(6.4)$ & $0.001 *$ & $0.001^{*}$ & $M>G>H$ \\
\hline HAMA & $0.3(0.9)$ & $10.8(5.3)$ & $18.9(8.0)$ & $0.001^{*}$ & $0.001^{*}$ & $M>G>H$ \\
\hline FT (times) & $30.4(9.4)$ & $29.3(9.9)$ & $31.1(9.0)$ & 0.980 & 0.915 & \\
\hline $\begin{array}{l}\text { VFT } \\
\text { (word counts) }\end{array}$ & $7.4(2.6)$ & $6.1(2.7)$ & $5.6(3.1)$ & $0.046^{*}$ & 0.120 & \\
\hline $\begin{array}{l}\text { DT } \\
\text { (word counts) }\end{array}$ & $8.1(3.4)$ & $6.9(2.4)$ & $5.4(2.4)$ & $0.016^{*}$ & $0.012^{*}$ & $\mathrm{H}>\mathrm{G}=\mathrm{M}$ \\
\hline $\begin{array}{l}\text { DTp } \\
\text { (word counts) }\end{array}$ & 8.7 (3.9) & $7.6(3.4)$ & $6.2(3.2)$ & 0.073 & 0.095 & \\
\hline \multicolumn{7}{|c|}{$\begin{array}{l}\text { Note: HC, healthy control subjects; GAD-MDD, generalized anxiety disorder without a comorbidity of major depressive disorder; GAD+MDD, } \\
\text { generalized anxiety disorder with a comorbidity of major depressive disorder; MEP, motor evoked potential; M1, primary motor cortex; HAMA, } \\
\text { Hamilton anxiety rating scale for anxiety symptoms; HAMD, Hamilton depression rating scale for depressive symptoms; FT, Finger tapping; VFT, } \\
\text { Verbal Fluency Testing; DT, dual tasks with simultaneous FT and VFT; DTp, DT under pretreated pressure }\end{array}$} \\
\hline \multicolumn{7}{|c|}{ aAll data are given as the mean (SD) if not otherwise specified; } \\
\hline \multicolumn{7}{|c|}{${ }^{*} p<0.05$ by ANOVA if not otherwise specified; post-hoc analysis by LSD. } \\
\hline
\end{tabular}


criteria of the initial enrollment also included those who were not native Chinese speakers and not familiar with Mandarin phonetic symbols. The study was performed in accordance with the Declaration of Helsinki and was approved by the Ethics Review Committee of the Taipei Veterans' General Hospital. Informed consent was completed by all participants prior to all experiments.

\section{- Clinical psychiatric and symptomatic evaluations}

All subjects underwent a detailed psychiatric, medical history-taking, and a diagnostic interview using the MINI. Handedness among subjects was confirmed using the Edinburgh Handedness Inventory [9]. With the exception of one patient in the GAD+MDD group being left-handed, all the recruited subjects were right-handed. Anxiety symptoms were rated by Hamilton rating scale of anxiety (HAMA) [10] and depression symptoms by Hamilton rating scale of depression (HAMD) [11]. After clinical evaluations, all subjects went through a Go/NoGo task, motor evoked potential evaluation, and fNIRS (mentioned below).

\section{- A neurocognitive test for attentional performance using right hand}

To rule out potential confounding from attentional deficits, a Go/No-Go task was also investigated before performing the fNIRS experiment. The Go/No-Go task we used in previous work [12,13] was adopted to test baseline attentional performance which requires the coordination of the hand and finger muscles. The reaction times (median and standard deviations) were recorded as index measurements for attentional performance using right hand and index finger. Other details please refer to the supplement.

\section{- Measuring cerebral activity using functional Near-Infrared Spectroscopy (fNIRS)}

A multichannel wearable fNIRS imaging system (NIRSport, NIRx Medical Technologies LLC, Glen Head, NY, USA) was used to simultaneously acquire dual-wavelength (760 and $850 \mathrm{~nm}$ ) signals. The fNIRS optodes, including 8 LED light sources and 8 detectors, were attached to participants' heads to monitor the hemodynamics of the bilateral prefrontal cortex (PFC), primary motor cortex (M1), and supplemental motor area (SMA) (Figure 1a). The fNIRS head cap was specifically designed in compatibility with the international 10-5 system, which defines standard surface positions for a human head with approximately $3.0 \mathrm{~cm}$ between any 2 adjacent positions [14]. In the source and detector arrangement, 14 effective source-detector channels were generated for monitoring local blood oxygenation (Figure 1a), with a sampling rate of $6.25 \mathrm{~Hz}$. During the signal acquisition, the time points for the beginning of each task condition were recorded. To enhance the signal-to-noise ratio and simplify the interpretation, the 14 effective channels were further categorized into 6 regions, including left PFC (channel 1), right PFC (channel 2), left M1 (channels 3 to 6), left SMA (channels 7 and 8), right SMA (channels 9 and 10), and right M1 (channels 11 to 14 ), by averaging the channels within the region (Figure 1a).

\section{- Experimental paradigm for fNIRS}

The experimental paradigm comprised of four task conditions, the finger taping (FT), verbal fluency testing (VFT), dual tasks (DT), and dual tasks under enhanced psychological pressure (DTp) (Figure 1a). Brain hemodynamic changes and task performance were recorded during the tasks. A block design with a random order of the first 3 conditions (FT, VFT, and DT) was applied. Each condition was repeated 3 times yielding a total of 9 testing blocks (block length 40 seconds) (Figure 1c). Three consecutive blocks of the DTp condition were then added after the above-mentioned 9 testing blocks and concatenated with one block of FT between the second and third block of DTp as a short switch of condition. A resting interval (for 40 seconds) was arranged between any two conditions. During the resting sessions, participants were asked to sit comfortably without any head or body motion until they heard the instruction for the coming tasking condition. All participants were native speakers who were capable of performing verbal fluency part of the tasks in a Chinese version.

\section{- Finger taping (FT)}

Participants were verbally instructed to perform a self-paced unilateral finger tapping (finger to thumb opposition movement) using their habitual hand until instructed to rest (approximate 40 seconds). Participants practiced the task to get a uniform rhythm of $1 \mathrm{~Hz}$ before the investigation.

\section{- Verbal fluency testing (VFT)}

Participants were asked to generate words starting with a Chinese phonetic symbol (e.g., /b(々)/, 

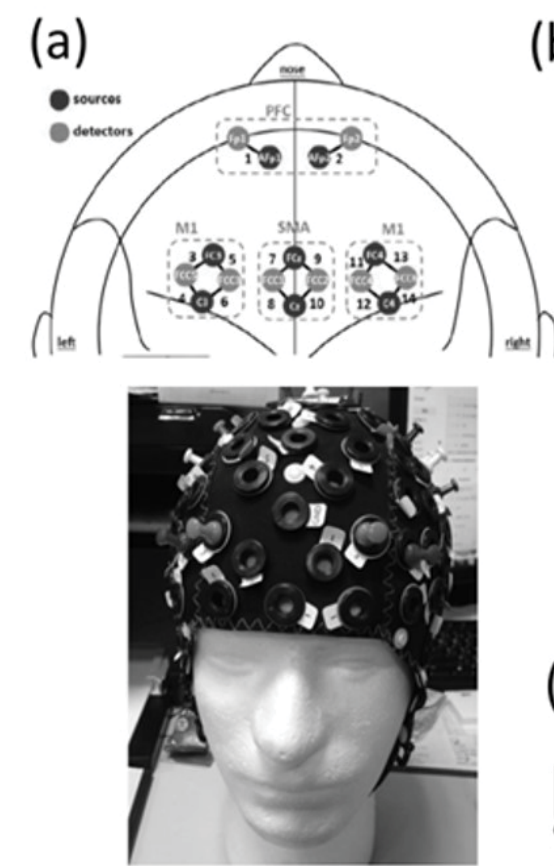

(b)

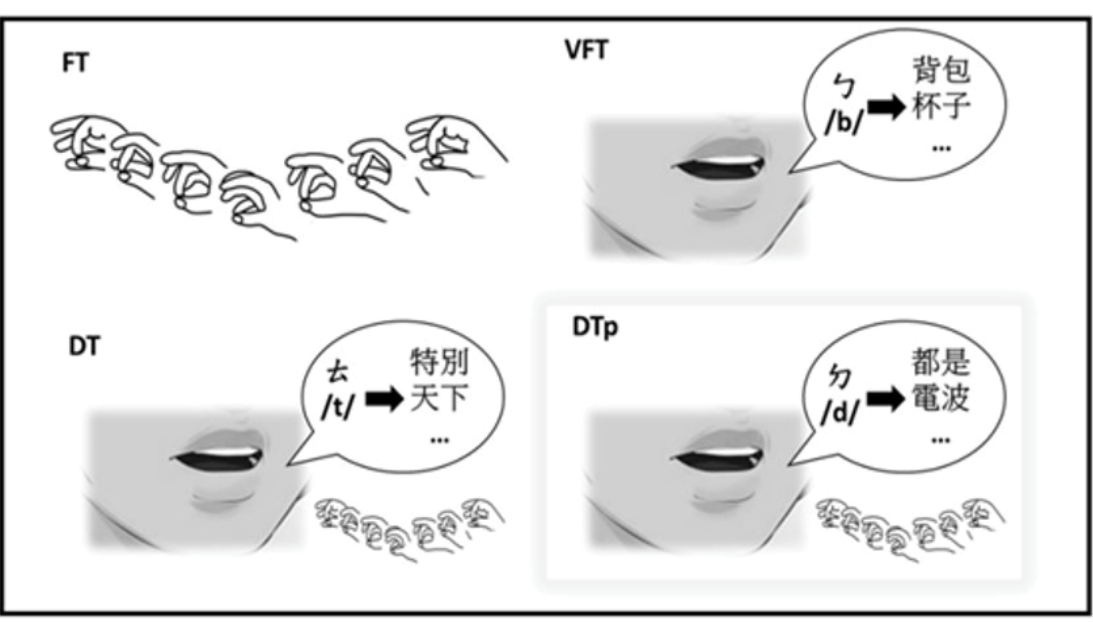

(c)

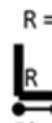

$R=$ rest for $40 \mathrm{sec}$

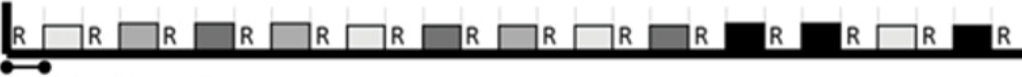

Block duration $=40 \mathrm{sec}$

Figure 1: Task paradigm and fNIRS measurement setting.

(a) configuration of measurement channels including sources and detectors for bilateral primary motor cortex (M1; channel 3-6 and 11-14). Supplementary motor area (SMA) and prefrontal cortical area (PFC) were also covered to be reference regions. Figure below showed a photograph for demonstration.

(b) four tasks used in the fNIRS, including finger tapping (FT), verbal fluency testing (VFT), dual tasks with simultaneous FT and VFT (DT), and DT with pretreated psychological pressure (DTp) by informing the participant before the task of his/her far below average performance so far and a need to do better in the coming session.

(c) A block design of the experimental paradigm with a random order.

$/ \mathrm{p}($ 女 $) /, / \mathrm{m}(\Pi) /, / \mathrm{f}(\sqsubset) /, / \mathrm{d}($ 分 $) /, / \mathrm{t}($ 古 $) / \ldots)$ as many as possible within 40 seconds. Word generation and counting were recorded during the task. To ensure sufficient compliance, each participant had adequate practice trials prior to the experiment.

\section{- Dual tasks (DT)}

Participants were instructed to perform FT and VFT simultaneously within a task session. Participants were asked to generate words as many as possible while continuing finger taping. The task period was approximately 40 seconds and ended with a resting instruction.

\section{- Dual tasks under pretreated psychological pressure (DTp)}

Right before the next session of DT, participants were intentionally informed that their performance in the previous DT sessions was far below average and encouraged to enhance their efforts on the task. This was done specifically to investigate the effect of DT challenge under extra mental stress. The task period was approximately 40 seconds and ended with a resting instruction.

\section{- fNIRS data analysis}

To estimate the signal-to-noise quality of a data channel, the relative coefficient of variation $(\mathrm{CV}$, in \%) was calculated for the raw signals at 760 and $850 \mathrm{~nm}$, which is a routine procedure for fNIRS measurement [15]. The data quality for five subjects (three patients and two healthy control subjects) was poor so their data were not included for further analyses. Data rejection based on 2 types of $\mathrm{CV}, \mathrm{CV}_{\text {chan }}$ and $\mathrm{CV}_{\text {trial' }}$, was used to reduce physical artifacts such as motioninduced instabilities and blood pressure-induced hemodynamics [16].

$\mathbb{C}=\frac{\sigma}{\mu} \times 100 \%$

where $\mu$ is the mean value and $\sigma$ is the standard deviation of the signal. $\mathrm{CV}_{\text {chan }}$ was calculated over the entire duration of the experiment (approximate 18 minutes) for each channel, and measurement channels with $\mathrm{CV}_{\text {chan }}>15 \%$ 
was rejected. The $\mathrm{CV}_{\text {trial }}$ was then obtained for 40 -second intervals of the individual trial block, and only trials for each remaining channel $\left(\mathrm{CV}_{\text {cha }}\right.$ $<15 \%$ ) with $\mathrm{CV}_{\text {trial }}<10 \%$ in both wavelengths were retained for subsequent analyses.

The remaining fNIRS signals were bandpassfiltered (low-cutoff frequency $0.01 \mathrm{~Hz}$ and high-cutoff frequency $0.05 \mathrm{~Hz}$ ) to eliminate the effects of heartbeat, respiration, and lowfrequency signal drifts for each wavelength [16]. The wavelet filtering was subsequently used to correct for the motion artifacts in each channel. The measured signal is assumed to be a linear combination of the physiological signal of interest (hemodynamics) and the motion artifacts. Since the hemodynamic response is much slower than motion artifacts (such as a spike artifact), the wavelet coefficients for the evoked response are anticipated to be a Gaussian probability distribution with zero mean and low variance whereas the outliers accounting for the motion artifacts. the outlying coefficients were eliminated (larger than a predefined threshold) before signal reconstruction using the inverse discrete wavelet to eliminate the corresponding motion artifacts. The removal threshold of the wavelet coefficient, $\alpha$, was set to 0.1 in this study [17]. The preprocessed signals were converted to concentration changes in oxygenated hemoglobin, $\Delta[\mathrm{HbO}]$, and deoxygenated hemoglobin, $\Delta[\mathrm{HbR}]$, using the modified BeerLambert law for each source-detector channel study [18]. Afterwards, the correlation-based signal improvement was employed to improve the signal quality based on the findings that brain activation involves $\Delta[\mathrm{HbO}]$ increases and $\Delta[\mathrm{HbR}]$ decreases at the activated cortical regions [19]. The relative changes in [HbO] and $[\mathrm{HbR}]$ concentrations (i.e., $\Delta[\mathrm{HbO}]$ and $\Delta[\mathrm{HbR}])$ at each time point were calculated based on a 5-second baseline (approximately 31 frames) and collected before proceeding with each task and then averaged over 3 repetitions for each condition.

Bilateral M1 was our primary region of interests (ROI), while bilateral SMA and PFC were also investigated as reference regions. The fNIRS signal preprocessing, including the bandpass filtering, motion artifact correction, and calculation of $\Delta[\mathrm{HbO}]$ and $\Delta[\mathrm{HbR}]$ were processed using the HOMER2 package [20]. Calculation of signal $\mathrm{CVs}$ and quantitative analyses were performed using home-made scripts developed on MATLAB (Mathworks, Natick, MA, USA).
- Increases of total hemoglobin $(\Delta[\mathrm{Hb}])$ as a key index for brain activation

To quantify the hemodynamic responses to each task condition, the areas under the curve (AUC) of $\Delta[\mathrm{HbO}]$ and $\Delta[\mathrm{HbR}]$ were calculated between the 5 to 30 seconds after task onset. Neuronal activation typically induces an increase of cerebral metabolic rate of oxygen with a larger compensatory increment of local cerebral blood flow based on neurovascular coupling [21]. The cortical activation related hemodynamic responses typically involves a rapid elevation of $\Delta[\mathrm{HbO}]$ and a lower-amplitude reduction in $\Delta$ $[\mathrm{HbR}]$ as confirmed by simultaneous measures of other neuroimaging techniques [22]. Total hemoglobin increases $(\Delta[\mathrm{Hb}])$ was calculated as the sum of $\Delta[\mathrm{HbO}]$ and $\Delta[\mathrm{HbR}]$ and were used as a proxy measure of the underlying brain activation and brain activity in response to four tasks (i.e., cortical responsiveness) was analyzed. It has been demonstrated in a detailed animal study that $\Delta[\mathrm{Hb}]$ is overall the most sensitive marker of cerebral blood flow changes comparing $\Delta[\mathrm{Hb}], \Delta[\mathrm{HbO}]$ and $\Delta[\mathrm{HbR}][23]$.

\section{n Motor Evoked Potentials (MEPs)}

To confirm that the channels 3 to 6 and channels 11 to 14 accurately covered the bilateral M1 controlling fingers, surface electromyography (EMG) recordings were made before the fNIRS paradigm. The EMG was recorded from the bilateral abductor pollicis brevis (APB) muscles by transcranial magnetic stimulation. The resting motor threshold (rMT, expressed as the percentage of maximum stimulator output) was also recorded and defined as the lowest intensity which produced an MEP of $>=50 \mu \mathrm{V}$ in 5 of 10 trials in the relaxed APB muscle [24]. Other details please refer to the supplement.

\section{Statistics}

SPSS 16.0 software (SPSS Inc., Chicago, IL) was used. Independent t-tests and analysis of variance (ANOVA) were used to compare the continuous variables (e.g., age, symptom ratings, word counts of VFT, DT, and DTp, and $\Delta[\mathrm{Hb}]$ ) between two and three independent groups, respectively. Chisquare test will be applied to compare categorical variables among groups. Statistical significance was set at a $\mathrm{p}<0.05$ with LSD for post-hoc comparisons. Before the Independent t-tests and ANOVA analysis, we used the Levene's test for homogeneity of variances to ensure homogeneity assumption. Where results from 
Levene's test were significant $(p<0.05)$, we used "equal variances not assumed" for correction in the Independent t-tests and Kruskal-Wallis test (non-parametric analysis) were applied for the 3 group comparisons. To eliminate potential influences of clinical ratings on M1 $\Delta[\mathrm{Hb}]$, repeated measures ANOVA (rmANOVA) was applied for comparing M1 $\Delta[\mathrm{Hb}]$ between DT, DTp, VFT, and VT, with age, sex, trait worry, anxiety and depressive symptoms being treated as covariates of no interests. Statistical significance was also set at a $\mathrm{p}<0.05$. Pearson's correlation was applied to study potential correlations between clinical symptoms and M1 $\Delta[\mathrm{Hb}]$. Receiver operating characteristic (ROC) curves were plotted for assessing the accuracy of predictions of illness from healthy control group by M1 $\Delta$ [Hb]. Area under the ROC curve (AUC) and the optimal cutoff with maximum sum of sensitivity and specificity were calculated [25].

\section{Results \\ - Clinical characteristics, cognitive profiles, and symptom ratings among groups}

The demographic data and baseline attentional function as measured by the Go/No-Go testing did not differ among the 3 groups (Table 1). GAD patients with or without MDD had no difference of illness duration (mean $(\mathrm{SD})=$ 13.0 (10.7) vs. 11.3 (11.9) years) (Table 1). In addition, the attentional function by the Go/ No-Go task and resting motor threshold by $100 \%$ motor evoked potentials (rMT) did not differ among three groups. All the recruited GAD patients were medication free, including $62.5 \%$ of them were drug-naive. For those who stopped medication uses $(n=13)$, the antidepressants included escitalopram $(n=8,15$ $45 \mathrm{mg} /$ day), agomelatine ( $\mathrm{n}=3,25 \mathrm{mg} /$ day), and sertraline $(\mathrm{n}=2,50 \mathrm{mg} / \mathrm{day})$; eight patients also stopped the anxiolytics, including alprazolam $(\mathrm{n}=1,0.5 \mathrm{mg} /$ day $)$, clobazam $(\mathrm{n}=3,5-10 \mathrm{mg} /$ day), and lorazepam $(\mathrm{n}=4,0.25-0.5 \mathrm{mg} /$ day $)$. GAD patients had higher HAMA and HAMD than HC (post-hoc Bonferroni for both, $\mathrm{p}<0.05$ : GAD+MDD > GAD-MDD > HC). Regarding the performance of $\mathrm{NIRS}$ tasks, GAD patients performed significantly worse on the VFT and DT tasks than the HC (Table 1).

\section{- fNIRS findings among groups}

The results of $\Delta[\mathrm{Hb}]$ during the four tasks in bilateral M1 are depicted in Figure 2, showing data from -5 to $50 \mathrm{sec}$ for visual inspection in detail. In almost all of the tasks, GAD had abnormally attenuated increases of $\Delta[\mathrm{Hb}]$ over bilateral M1 than HC (Figure 2 and Table 2). The pattern of the attenuated M1 cortical responsiveness in GAD was robust and was observed even in pure motor task (FT) and pure cognitive tasks (VFT). If being more conservative and applying more rigid statistical criteria, left M1 $\Delta[\mathrm{Hb}]$ in response to VFT and DTp remained statistically significant after correcting for multiple comparisons (Table 2, marked in open stars). Such pattern was not observed in the reference regions including PFC and SMA (Figure S1). No statistical significance was found in all M1 $\Delta[\mathrm{Hb}]$ findings between GAD with and without MDD. Pearson's correlational analysis showed no significant correlation of the M1 $\Delta[\mathrm{Hb}]$ and clinical symptoms and the performance of fNIRS tasks. Thus the attenuated M1 responsiveness could be a trait characteristic of GAD, independent of anxiety or depressive symptoms.

To further validate the M1 $\Delta[\mathrm{Hb}]$ as the most prominent variables differentiating GAD from $\mathrm{HC}$, repeated-measure ANCOVA was done with the elimination of age, sex, worry, anxiety, and depression and we compared M1 $\Delta[\mathrm{Hb}]$ between DTp, DT, VFT, and FT among GAD and HC groups (Figure S2). The results revealed a significant main effect of group on bilateral M1 $\Delta[\mathrm{Hb}]$ results (left M1: $F=11.026, p=0.002$, observed power $=0.903$; right $\mathrm{M} 1: \mathrm{F}=9.843$, $p=0.003$, observed power $=0.869)$, indicating that GAD had significantly lower M1 $\Delta[\mathrm{Hb}]$ across all tasks than $\mathrm{HC}$ after controlling out the potential influences of age, sex, and clinical symptoms.

\section{- Predicting GAD patients from HC by M1 $\Delta[\mathrm{Hb}]$ during 4 tasks}

The area under the ROC curves (AUC) for bilateral M1 $\Delta[\mathrm{Hb}]$ in response to four different tasks were demonstrated in Figure 3. The best predicting value was the AUC of left M1 $\Delta[\mathrm{Hb}]$ during VFT $(\mathrm{p}<0.001)$ and the second one was that of left M1 $\Delta[\mathrm{Hb}]$ during DTp ( $=0.004)$. For left M1 $\Delta[\mathrm{Hb}]$ during VFT, setting the threshold at 8.206 by using the optimal cutoff with maximum sum of sensitivity and specificity, the sensitivity and specificity given to accurately predict $\mathrm{HC}$ from GAD are $80 \%$ and $75 \%$, respectively. Since anxiety symptoms are common in the modern society, these results evidently indicate and show that M1 $\Delta[\mathrm{Hb}]$ 
Attenuated Motor Cortical Responsiveness to Motor and Cognitive Tasks in Generalized Anxiety Disorder

FT

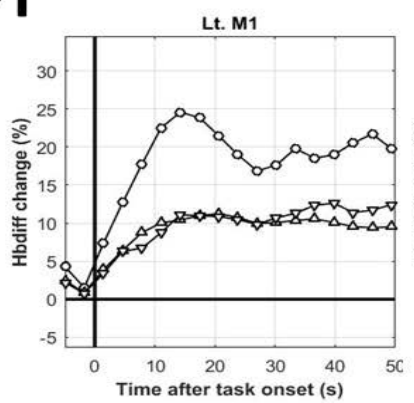

DT

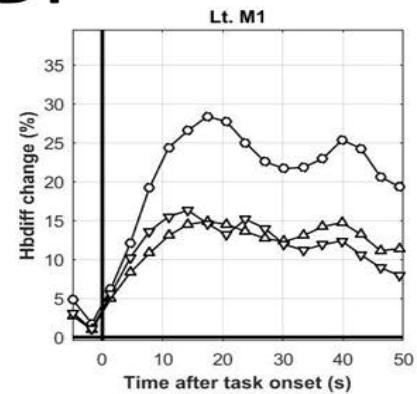

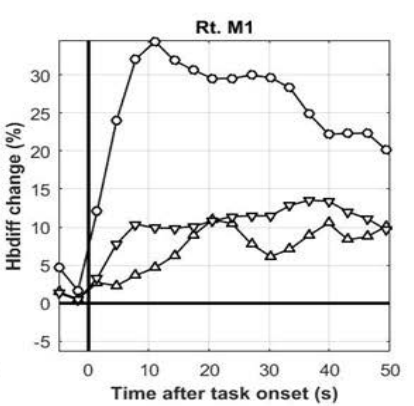

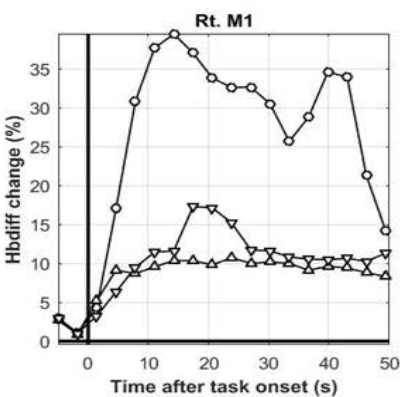

VFT

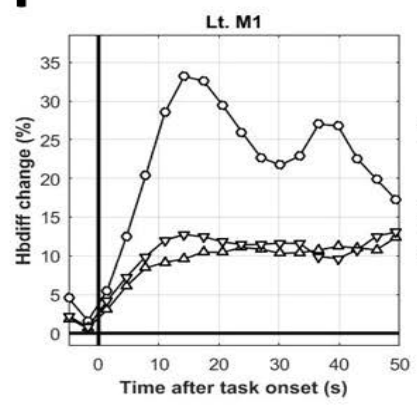

DTp

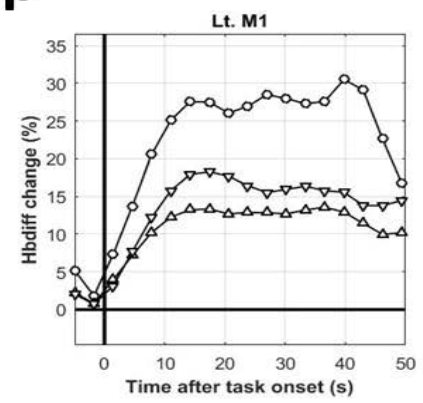

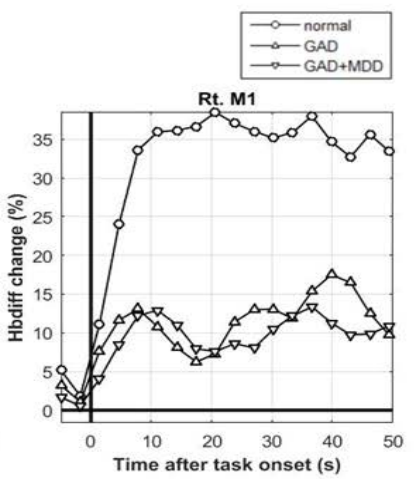

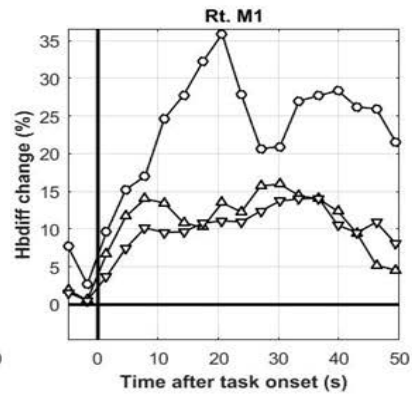

Figure 2: Block-averaged time courses of fNIRS M1 $\Delta[\mathrm{Hb}]$ changes in response to four tasks among 3 groups, consistently showing attenuated $\mathrm{M} 1 \Delta[\mathrm{Hb}]$ increases in GAD patients with or without MDD as compared to healthy control subjects (normal).

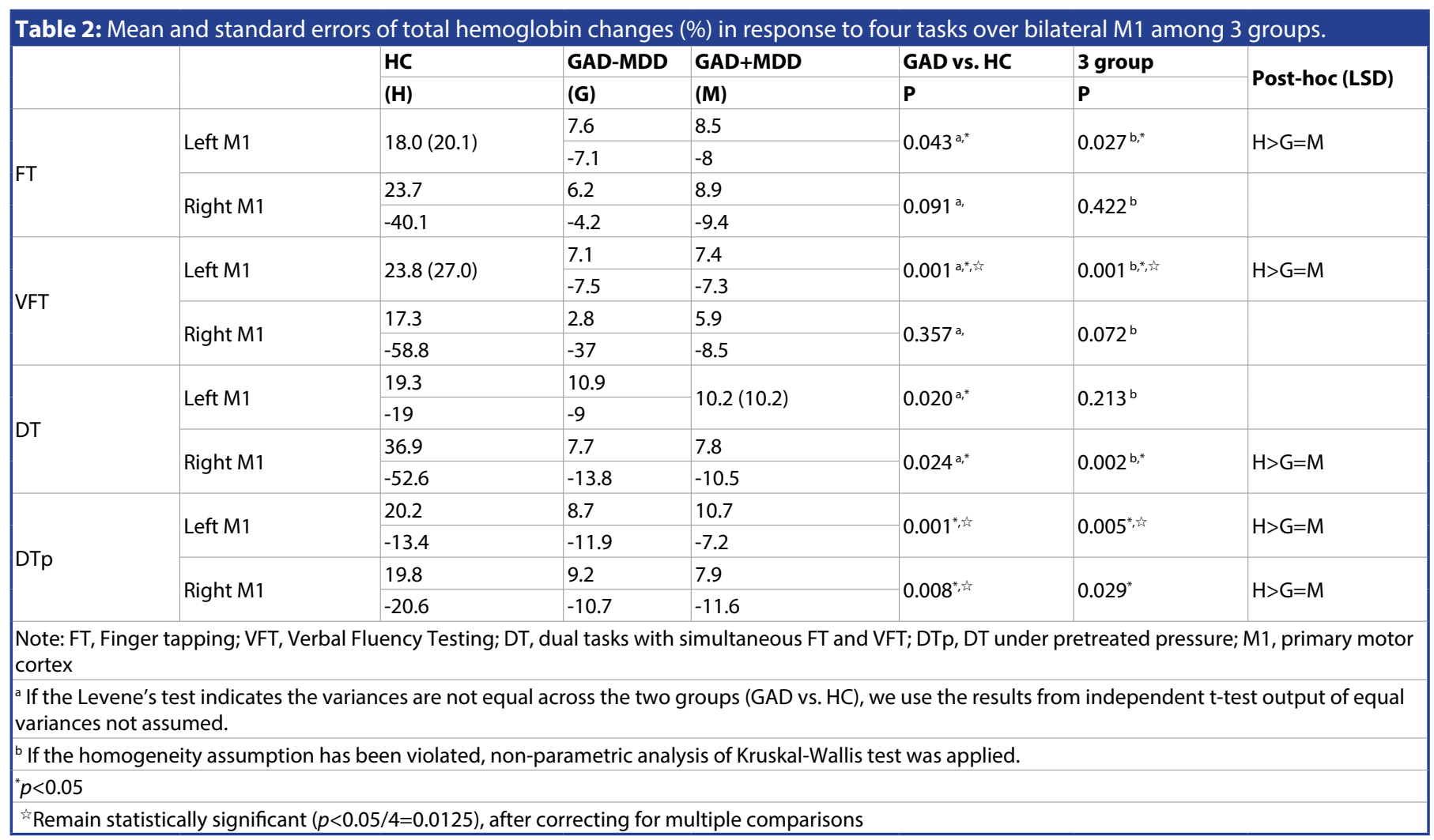

in responses to cognitive load tasks (i.e., VFT biomarker in predicting illness from healthy and DTp) could be used as a highly efficient normal subjects. 
FT

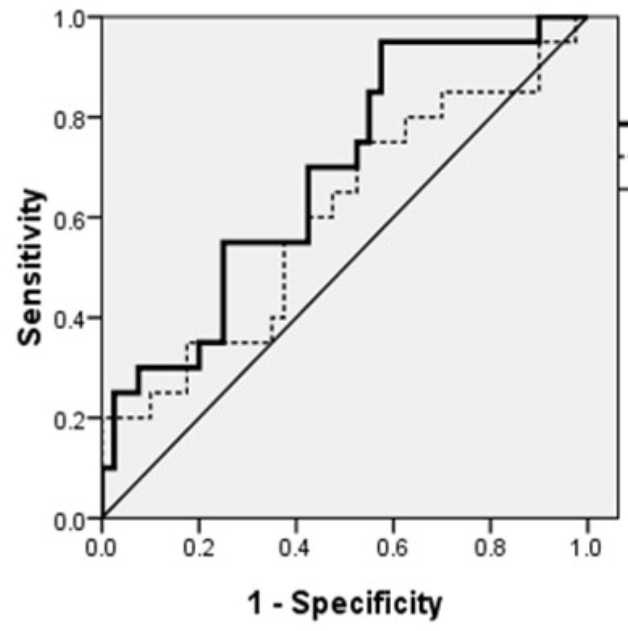

DT

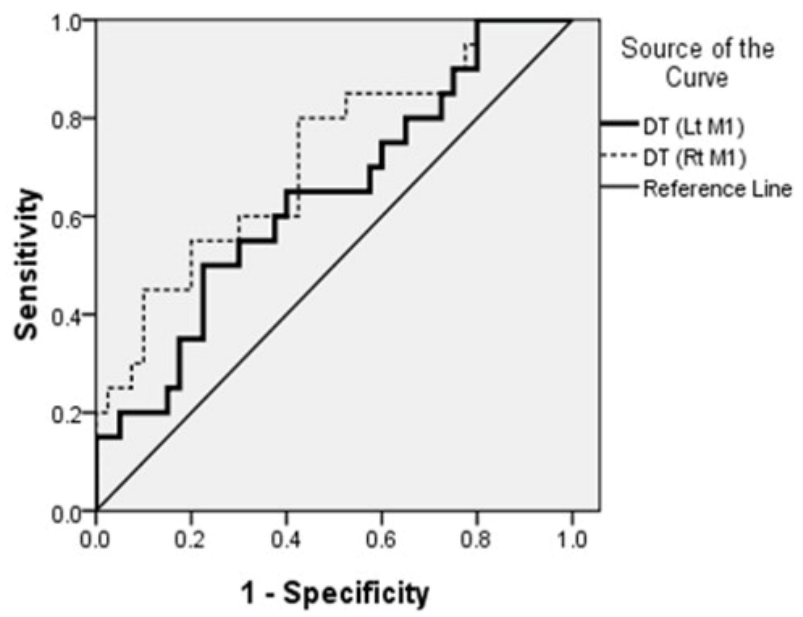

VFT Roc Curve

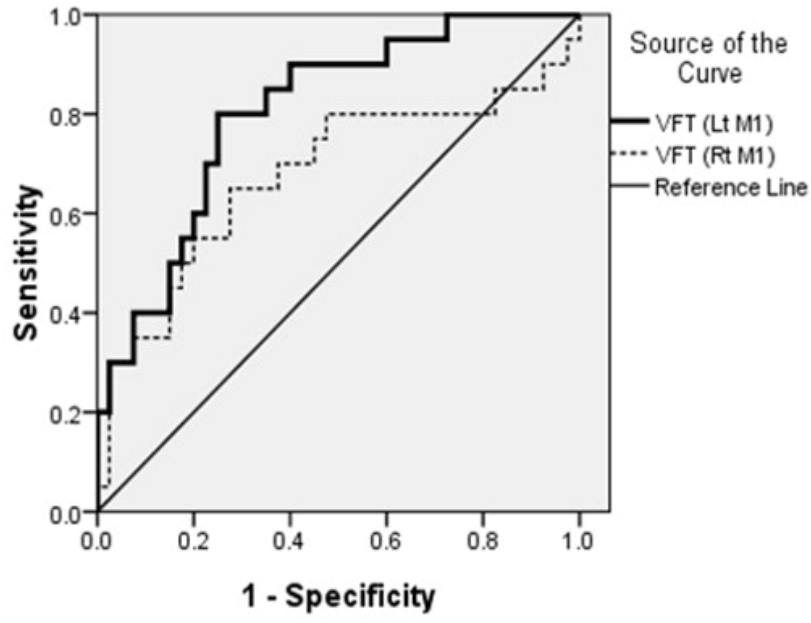

DTp Roc Curve

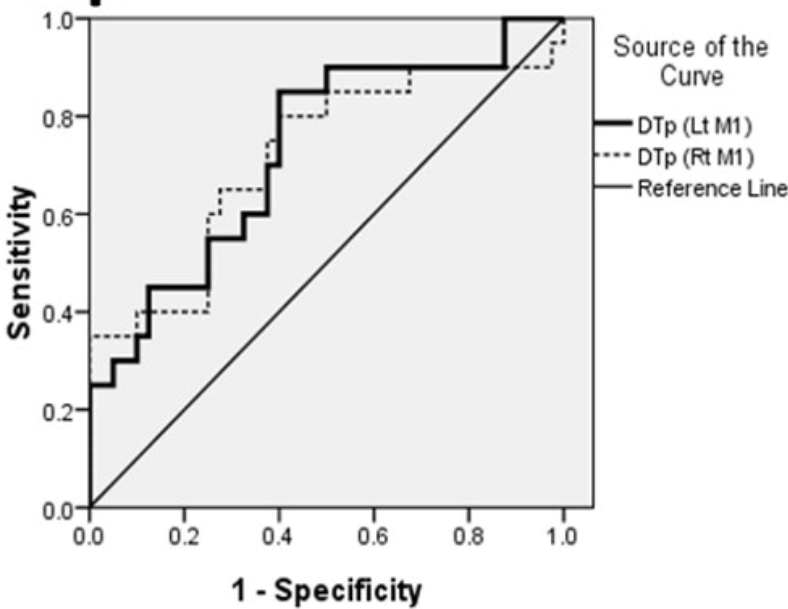

Figure 3: Receiver operating characteristic (ROC) curves. The left and right M1 $\Delta$ [Hb] in response to FT, VFT, DT, and DTp have good sensitivity/ specificity in predicting a diagnosis of GAD from healthy controls (all $\mathrm{p}<0.05$ except right $\mathrm{M} 1 \Delta$ [Hb] in response to FT). During FT, area under the ROC curve for left M1 $\Delta[\mathrm{Hb}$ ] (thick solid line) was 0.685 and for right (dashed line) was 0.601 . During VFT, area under the ROC curve for left $\mathrm{M} 1 \Delta$ [Hb] (thick solid line) was 0.805 and for right (dashed line) was 0.708 . During DT, area under the ROC curve for left M1 $\Delta$ [Hb] (thick solid line) was 0.640 and for right (dashed line) was 0.718. During DTp, area under the ROC curve for left M1 $\Delta[\mathrm{Hb}$ ] (thick solid line) was 0.729 and for right (dashed line) was 0.716 . The best predicting value was left $M 1 \Delta[\mathrm{Hb}]$ in response to VFT $(p<0.0001)$ and the second one was left M1 $\Delta[\mathrm{Hb}]$ in response to DTp ( $p=0.004)$.

\section{Discussion}

The present study is the first that utilized multiple tasks in combination with fNIRS and specifically examined motor cortical function in patients with generalized anxiety disorders (GAD). We found that attenuated M1 cortical responsiveness as a characteristic feature of GAD (Table 2) (Figure 2) and the range of decreases of the M1 cortical activations in patients with GAD was from $-45.3 \%$ to $-79.0 \%$ across tasks. In addition, such attenuated M1 responsiveness cannot be explained by medication (all patients medication free and fifty-five percent drug- naive) and did not differ between GAD patients including both with and without a comorbidity of MDD. The severity of anxiety and/or depressive symptoms was not associated with the task-induced changes of M1 activities and was not a confounding factor biasing our findings since the repeated measure ANCOVA controlling out clinical symptoms consistently revealed the attenuated M1 cortical activations across tasks in GAD patients versus healthy control subjects (Figure S2). Furthermore, the ROC analysis revealed that left M1 $\Delta[\mathrm{Hb}]$ in response to cognitive tasks, especially the pure 
cognitive task (VFT), has good sensitivity and specificity in predicting a diagnosis of GAD from healthy controls (Figure 3).

As hypothesized, we found that abnormal function in the primary motor cortex could be a characteristic trait feature of GAD. The finding of abnormal M1 function in GAD was in line with previous findings. For example, we recently measured cortical excitability by pairedpulse transcranial magnetic stimulation and found that GAD patients had significantly lower intracortical facilitation in bilateral M1 [26]. In addition, research using resting-state functional MRI had revealed that GAD patients had significantly decreased functional connectivity to amygdala from many cortical regions, including bilateral M1 [5]. This finding of attenuated M1 responsiveness partially explains the fatigue and muscle tension in GAD patients which has been repeatedly reported as the most specific symptom for a diagnosis of GAD from studies that utilized a large sample size [27,28]. For example, by investigating community subjects with GAD, Faravelli et al. recently reported that only two of the GAD diagnostic symptoms (excessive worries and muscle tension) showed a specific association with the diagnosis itself, while the other symptoms (e.g., concentration problem and fatigue) were found to be more prevalent in depression than in GAD. The odd ratio indicating the specific association of muscle tension and GAD diagnosis was reported to be as high as 10.18 [28]. Despite the present study found no association between clinical rating symptoms and $\mathrm{M} 1 \Delta[\mathrm{Hb}]$, the potential connection between the abnormal motor function and symptoms in the muscle system should not be entirely ruled out as most of our GAD patients $(82.5 \%)$ reported the symptom of muscle tension over the past six months. As a next step, future studies directly evaluating the severity of muscle tension are vital.

The mechanism underlying the attenuated motor cortical responsiveness remains unknown. However, resting motor threshold of $100 \%$ MEP has been found to positively reflect regional M1 neuronal activity at rest [7]. Since an increase of the resting motor threshold in the GAD groups was found (Table 1), our finding of the attenuated M1 cortical activations in GAD may therefore be attributed to higher M1 activity at baseline and reduced reservoir capacity of M1 for muscle performance and control. The changes in the baseline motor threshold could be also related to the chronic anxious state, since worry has been found to significantly change and facilitate M1 activities [6]. Therefore, we speculate that the observation of attenuated M1 cortical activations in GAD may involve a compensatory downregulation of the muscle-controlling motor system for combating M1 activities induced by long-term worry and pathological anxiety.

The findings of M1 $\Delta[\mathrm{Hb}]$ changes during VFT were not unexpected. It has been reported that the cortical activations during vocalization tasks in the hand area were the result of functional connectivity between hand and vocalizing brain regions, instead of an underlying vascular overflow mechanism from the adjacent speech processing or face/mouth areas [29]. Moreover, spatial resolution of fNIRS is not so high that the delineation of the optodes (i.e., sources and detectors (Figure 1), around the bilateral M1 channels may also detect signals from brain regions controlling face, mouth, or other speechprocessing structures [30].

The main strengths of the present study included a use of fNIRS that avoids unwanted stress related to the imaging procedure (e.g., lying in a tunnel, large sounds, radiation exposure) in an easily-anxious population, a recruitment of medication-free GAD patients with and without a comorbidity of MDD (not one patient population and a consistent finding of M1 $\Delta$ [Hb] in both GAD groups), utilizing multiple tasks that pinpointed to the motor cortex, and the use of pre-task assessment of attentional function and resting motor activities by the Go/No-Go task and motor-evoked potentials, respectively. However, the present study also has some limitations. Firstly, the experiment design was cross-sectional and whether or not the motor cortical deficits could be normalized after adequate medication treatment requires further study. Secondly, our design was not aimed at biomarkers differentiating pure GAD patients from those with a comorbidity of MDD and the present study specifically focused on bilateral motor cortices. The finding of no difference of the M1 $\Delta[\mathrm{Hb}]$ between GAD with and without MDD should not be conclusive that GAD with and without a comorbidity of MDD have the same brain dysfunction. On the contrary, although both GAD-MDD and GAD+MDD groups demonstrated similar abnormalities in the $\mathrm{M} 1$, their $\Delta[\mathrm{Hb}]$ responses differ in other brain regions. For example, the preliminary data from the present study revealed that $\Delta[\mathrm{Hb}]$ increases of left supplementary motor area during VFT were higher in GAD-MDD than that in 
GAD+MDD $(\mathrm{p}<0.05)$. Further exploration is required to disentangle biomarkers that help differentiate a comorbidity of MDD in GAD.

\section{Conclusion}

By utilizing multiple tasks in combination with fNIRS, the present study was the first to support that attenuated M1 cortical responsiveness could have a diagnostic value in clinical settings and in fact be a characteristic trait feature of GAD, irrespective of a comorbidity of MDD or the severity of anxiety and depression symptoms.

\section{- Financial Disclosures and Conflict of Interests}

The study was sponsored by grants from Taipei Veterans General Hospital (VGHUST102-G4-3-1, V104E9-003, and V104B-002) and Ministry of Science and Technology MOST 106-2314-B-038024-MY2 and TMU103-AE1-B02. None of the aforementioned funding organizations had any role in the study design, data collection, analysis, interpretation of result, writing of the report, and the ultimate decision to submit the paper for publication. None of the authors in this study had any conflict of interest to declare.

\section{References}

1. Tully PJ, Cosh SM, Baune BT. A review of the affects of worry and generalized anxiety disorder upon cardiovascular health and coronary heart disease. Psychol. Health. Med 18(6), 627-644 (2013).

2. Mochcovitch MD, da Rocha Freire RC, Garcia RF, et al. A systematic review of fMRI studies in generalized anxiety disorder: evaluating its neural and cognitive basis. J. Affect. Disord 167(1), 336-342 (2014).

3. Fonzo GA, Etkin A. Affective neuroimaging in generalized anxiety disorder: an integrated review. Dialogues. Clin. Neurosci 19(1), 169-179 (2017).

4. Feinstein JS, Adolphs R, Damasio A, et al. The human amygdala and the induction and experience of fear. Curr. Biol 21(1), 3438 (2011).

5. Etkin A, Prater KE, Schatzberg AF, et al. Disrupted amygdalar subregion functional connectivity and evidence of a compensatory network in generalized anxiety disorder. Arch. Gen. Psychiatry 66(12), 13611372 (2009).

6. Oathes DJ, Bruce JM, Nitschke JB. Worry facilitates corticospinal motor response to transcranial magnetic stimulation. Depress. Anxiety 25(11), 969-976 (2008).

7. Li CT, Lu CF, Lin HC, et al. Cortical inhibitory and excitatory function in drug-naive generalized anxiety disorder. Brain. Stimul 10(3), 604-608 (2016).

8. Ferrari M, Quaresima V. A brief review on the history of human functional near-infrared spectroscopy (fNIRS) development and fields of application. Neurolmage 63(1), 921-935 (2012).

9. Oldfield RC. The assessment and analysis of handedness: the Edinburgh inventory. Neuropsychologia 9(1), 97-113 (1971).

10. M. The assessment of anxiety states by rating. Br. J. Med. Psychol 32(1), 50-55 (1959).
11. Hamilton M. A rating scale for depression. J. Neurol. Neurosurg. Psychiatry 23(1), 56-62 (1960). Feinstein

12. Li CT, Lin CP, Chou KH, et al. Structural and cognitive deficits in remitting and non-remitting recurrent depression: a voxel-based morphometric study. Neuroimage 50(1), 347-356 (2010).

13. Li CT, Su TP, Wang SJ, et al. Prefrontal glucose metabolism in medication-resistant major depression. Br. J. Psychiatry 206(4), 316-323 (2015).

14. Oostenveld R, Praamstra P. The five percent electrode system for high-resolution EEG and ERP measurements. Clin. Neurophysiol 112(4), 713-719 (2001).

15. Schmitz CH, Klemer DP, Hardin R, et al. Design and implementation of dynamic near-infrared optical tomographic imaging instrumentation for simultaneous dual-breast measurements. Applied. Optics 44(1), 2140-2153 (2005).

16. Piper SK, Krueger A, Koch SP, et al. A wearable multi-channel fNIRS system for brain imaging in freely moving subjects. Neurolmage 85(1), 64-71 (2014).

17. Molavi B, Dumont GA. Wavelet-based motion artifact removal for functional near-infrared spectroscopy. Physiol. Meas 332(1), 259 (2012)

18. Kocsis $L$, Herman $P$, Eke A. The modified Beer-Lambert law revisited. Phys. Med. Biol 51(5), N91 (2006).

19. Cui X, Bray S, Reiss AL. Functional near infrared spectroscopy (NIRS) signal improvement based on negative correlation between oxygenated and deoxygenated hemoglobin dynamics. Neuroimage 49(4), 3039-3046 (2010).

20. Huppert TJ, Diamond SG, Franceschini MA, et al. HomER: a review of time-series analysis methods for near-infrared spectroscopy of the brain. Appl. Opt 48(1), D280-D298 (2009).
21. Ances BM, Wilson DF, Greenberg JH, Detre JA. Dynamic changes in cerebral blood flow, $\mathrm{O} 2$ tension, and calculated cerebral metabolic rate of $\mathrm{O} 2$ during functional activation using oxygen phosphorescence quenching. J. Cereb. Blood. Flow. Metab 21(5), 511-516 (2001).

22. Hock C, Villringer K, Muller-Spahn F, et al. Decrease in parietal cerebral hemoglobin oxygenation during performance of a verbal fluency task in patients with Alzheimer's disease monitored by means of near-infrared spectroscopy (NIRS)--correlation with simultaneous rCBF-PET measurements. Brain. Res 755(1), 293-303 (1997).

23. Hoshi Y, Kobayashi N, Tamura M. Interpretation of near-infrared spectroscopy signals: a study with a newly developed perfused rat brain model. J. Appl. Physiol 90(1), 16571662 (1985).

24. Kujirai T, Caramia MD, Rothwell JC, et al. Corticocortical inhibition in human motor cortex. J. Physiol 471 (1), 501-519 (1993).

25. Soreide K, Korner H, Soreide JA. Diagnostic accuracy and receiver-operating characteristics curve analysis in surgical research and decision making. Ann. Surg 253(1), 27-34 (2011).

26. Li CT, Juan CH, Su TP, et al. Transcranial Magnetic Stimulation-Derived Motor Parameters and Regional Glucose Metabolism of Motor Cortex. J. Neurol. Neurophysiol 7(1), 1-8 (2016).

27. Joormann J, Stober J. Somatic symptoms of generalized anxiety disorder from the DSMIV: associations with pathological worry and depression symptoms in a nonclinical sample. J. Anxiety. Disord 13(5), 491-503 (1999).

28. Faravelli C, Castellini G, Benni L, et al. Generalized anxiety disorder: is there any specific symptom? Comprehensive psychiatry 53(1), 1056-1062 (2012).

29. Lo YL, Zhang HH, Wang CC, et al. Correlation of near-infrared spectroscopy and 
Attenuated Motor Cortical Responsiveness to Motor and Cognitive Tasks in Generalized Anxiety

Research Disorder

transcranial magnetic stimulation of the motor cortex in overt reading and musical tasks. Motor. Control 13(1), 84-99 (2009).
30. Toronov V, Webb A, Choi JH, et al. Investigation of human brain hemodynamics by simultaneous near-infrared spectroscopy and functional magnetic resonance imaging. Med. Phys 28(4), 521-527 (2001). 